EPiC Series in Computing
Volume 58, 2019, Pages 310-316
Proceedings of 34th International Confer-
ence on Computers and Their Applications

\title{
Predicting Cow's Delivery Using Movement and Position Data Based on Machine Learning
}

\author{
Yusuke Ono, Ryo Hatano, Hayato Ohwada and Hiroyuki Nishiyama \\ Department of Industrial Administration, Tokyo University of Science \\ 7417604@ed.tus.ac.jp
}

\begin{abstract}
One of the major problem farmers face is that of a parturition accident. A parturition accident result in the death of the calf when the cow gives birth. In addition, it reduces the milk yield. The farmer must keep the cow under close observation for the last few days of pregnancy.

A novel method to predict a cow's delivery time automatically using time-series acceleration data and global position data by machine learning is proposed. The required data was collected by a small sensor device attached to the cow's collar. An inductive logic programming (ILP) method was employed for a machine learning model as it can generate readable results in terms of a formula for first-order logic (FOL). To apply the machine learning technique, the collected data was converted to a logical form that includes predefined predicates of FOL. Using the obtained results, one can classify whether the cows are ready for delivery.

Data was collected from 31 cows at the NAMIKI Dairy Farm Co. Ltd. Using the method described above, 130 readings were obtained. The five-fold cross-validation process verified the accuracy of the model at $56.79 \%$.
\end{abstract}

\section{Background}

A parturition accident of a cow is known as a serious problem for farmers. It is important for farmers to have a cow giving birth to a live calf. However, when she gives birth to a calf, and a parturition accident occurs, which could lead to the death of both the calf and the cow. Furthermore, this could result in a reduction in the milk yield. Therefore, a parturition accident can cause heavy losses for farmers and they need to avoid such accidents as much as possible.

The most important cause of a parturition accident is the difficult delivery of a cow. To prevent a difficult delivery, the farmer must to assist the cow during the delivery process. However, there is no visible indication of when a cow is ready to give birth. It is known that the important indicative signs of delivery are changes in the food intake of a cow and/or ingredients of her milk. A cow starts to give birth to the calf a few days after showing these signs. It is not only difficult to notice the indicative signs but also difficult to predict when the actual delivery process may start. The farmer must make a tremendous effort to avoid overlooking the indications. 
To overcome this issue, we aim to detect the cows' delivery time automatically using machine learning. A sensor device is attached to the cow's collar, and it collects the time-series acceleration data and global position data. A set of readable rules that can predict the delivery of a cow is generated using the inductive logic programming (ILP) method, which is logic-based supervised machine learning. As ILP rules can be regarded as a set of formulas of the firstorder logic (FOL), the status of a cow about to give birth can be predicted accurately through this method. It contributes to improving production efficiency and leads to a decrease in the farmers' work burden.

\section{Related Works}

Till date, relatively less studies have been conducted in applications of machine learning of dairy farming. Caraviello et al. [1] predicted whether a cow's insemination will succeed using the Alternating Decision Tree (ADTree) [2] in 2006. They used 300 or more features collected from cows that had been milked for a 150 days $^{1}$. The accuracy of their model was verified using the ten-fold cross validation method and was observed to be $75 \%$ accurate. Mammadova and Keskin [4] predicted mastitis in cows using support vector machine (SVM) in 2013. They employed the cow's milk yield and status of the cow's breast as parameters for readings. ${ }^{2}$ The sensitivity and the specificity of their model was $89 \%$ and $92 \%$, respectively. Grzesiak et al. [3] predicted cow's milk yield using an artificial neural network in 2006.

In their study, the proposed method showed a lower root mean square (RMS) and a higher coefficient of determination than the existing studies. Similarly, Saleh et al. [7] predicted the cows' reproductive value in 2012. They observed a correlation between milk yield, fat mass, and reproductive value using artificial neural network and neurofuzzy learning [6] and that value exceeded 0.9 .

\section{The Method for Predicting a Cow's Delivery}

In this study, data was collected from a sensor device and the cow's delivery time was predicted by (supervised) machine learning. The method is given as follows: Raw data was collected from small sensor devices attached to the collars of a group of cows. The data was processed to identify the features and label them for binary classification. The cow reaching delivery stage within a preordained time period was classified as positive class whereas one that did not reach the delivery stage in the given time, was classified as negative class. Finally, the ILP model was used to verify the accuracy of the results. As ILP generates a set of readable rules in terms of FOL formulas as a model, convenient rules that can predict the delivery of a cow can be generated.

\subsection{Specification of The Sensor Device}

To obtain a model for predicting the delivery, raw sensor data was collected using an appropriate sensor device. In this study, the time-series three-dimensional acceleration data and global positioning data was used to construct the raw data set. To collect such data, the device "LL1000" by Life Laboratory Co., Ltd. was used (Figure 1). As the size of the device is 30

\footnotetext{
${ }^{1}$ In total, they used 17,587 results from 9,516 cows.

${ }^{2}$ They attached electrodes to the cow's breast and collected electronic signals as raw data for machine learning.
} 
$\mathrm{mm} \times 60 \mathrm{~mm} \times 10 \mathrm{~mm}$, it was small enough to attach to the cow's collar. The device measures acceleration, atmospheric pressure, temperature of the environment, and global position approximately three times per second and transfers the readings to other devices via Bluetooth. The transferred data is stored in a comma separated file (CSV file) on our computer. Figure 2 shows an example of the CSV file where columns give values for RSSI, ID, and the $x, y$, and $z$ axes represent the signal strength, sensor's ID number, and the acceleration, respectively.

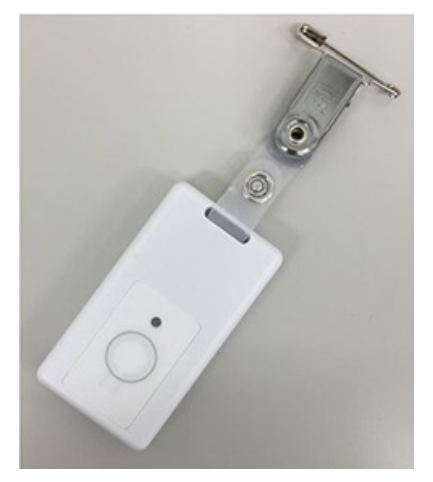

Figure 1: Sensor device LL1000

\begin{tabular}{|l|l|r|r|r|r|r|r|}
\hline \multicolumn{1}{|c|}{ A } & \multicolumn{1}{|c|}{ B } & \multicolumn{1}{c|}{ C } & D & \multicolumn{1}{c|}{ E } & \multicolumn{1}{c|}{ F } \\
\hline 1 & Date and Time & RSSI & ID & Xacc & Yacc & Zacc \\
\hline 2 & $2018-02-02 T 10: 49: 06.627+09: 00$ & -95 & 1 & 0.425 & 0.953 & 0.971 \\
\hline 3 & $2018-02-02 T 10: 49: 01.560+09: 00$ & -98 & 1 & 0.456 & -0.527 & -1.196 \\
\hline 4 & $2018-02-02 T 10: 49: 29.763+09: 00$ & -95 & 1 & 0.131 & 0.922 & -0.373 \\
\hline 5 & $2018-02-02 T 10: 50: 10.052+09: 00$ & -100 & 1 & 0.128 & 0.919 & -0.386 \\
\hline
\end{tabular}

Figure 2: Example of a CSV File of raw sensor data

\subsection{Making Features}

In this study, six types of features were obtained from the raw sensor data. Table 1, shows the features used in this method. From the acceleration data for each $x, y$, and $z$-axis, the norm was calculated for each axis (i.e. $\sqrt{a_{x}^{2}+a_{y}^{2}+a_{z}^{2}}$ ). When the sensor leans or tips over, the direction in which gravitational acceleration is applied changes. We use the sum of squares and prevent noise. The average and standard deviation was calculated based on the divided time-series acceleration data. The time-series data was divided into 10-min and 1-h intervals, respectively, in this study. Cows do not make intense movements in a short time. Therefore, it is too short to divide in 1-minute or shorter increments. The travel distance (Euclidean distance) was obtained from the global positioning data, at intervals of $10 \mathrm{~min}$ and $1 \mathrm{~h}$. That is, given a current time $t$ and a latitude lat and a longitude lon in a global positioning data, we define the travel distance by:

$$
\sqrt{\left(\text { lat }_{t}-\text { lat }_{t-1}\right)^{2}+\left(\operatorname{lon}_{t}-\operatorname{lon}_{t-1}\right)^{2}} .
$$


It should be noted that $t-1$ is $10 \mathrm{~min}$ or $1 \mathrm{~h}$ before the current time. The total travel distance is the sum of accumulated distance in the raw data.

Table 1: Types of features

\begin{tabular}{|l|l|l|}
\hline Acceleration $\begin{array}{l}\text { Average } \\
\text { Standard Deviation }\end{array}$ & $10 \mathrm{~min}$ & $1 \mathrm{~h}$ \\
\cline { 2 - 3 } & $10 \mathrm{~min}$ & $1 \mathrm{~h}$ \\
\hline Total Travel Distance & $10 \mathrm{~min}$ & $1 \mathrm{~h}$ \\
\hline
\end{tabular}

\subsection{Labeling of Features}

As mentioned before, features obtained from the raw sensor data were separated into the positive and the negative class, based on a predetermined time value $n$. To the best of our knowledge, the time taken by a farmer to prepare for the delivery of a cow is approximately $2 \mathrm{~h}$. In this study, the time is represented as, $n=2$, but this parameter will be more precisely determined in further studies.

\subsection{Inductive Logic Programming}

ILP is one of the machine learning approaches for developing inductive reasoning on first order predicate $\operatorname{logic}^{3}$. In this method, predicate logic is used to resolve classification problems [8]. Under the ILP approach, FOL can be used as a knowledge expression language to treat the relationship between individuals. Hence, multiple time-series data and mixed qualitative data is presented as the discrete expression of background knowledge.

Inductive reasoning is the inference which can ask for a generalized hypothesis from some specialized cases. Hence, an algorithm that can find relational and logical patterns between data (i.e. FOL-formulas) using inductive reasoning, corresponds to a learning or training algorithm of a model where the obtained model (so called rules) is written in terms of a set of FOL-formulas that may contain a generalized hypothesis ${ }^{4}$. We emphasize that the model is easy to understand for most people since it is written in terms of FOL formulas. As in a usual supervised machine learning model, the obtained model can be used to solve various classification problems.

\subsubsection{Features in Logical Form}

In order to apply the ILP approach, it is necessary to convert the data generated in the experiment, to a logical form. Thus, we need to convert the six features mentioned in Section 3.2 in the form of FOL-formulas. In this study, we define the following 8 predicates.

1. moveave600(Time,Level1-8)

Average of acceleration at 10 -min intervals.

2. moveave3600(Time,Level1-8)

Average of acceleration at 1-h intervals.

\footnotetext{
${ }^{3}$ It is known that ILP covers not only FOL but also other types of logic. Propositional logic is another example, as a model of decision tree can be regarded as a formula of ILP.

${ }^{4}$ More precisely, the obtained model is written in an extended form of logic programming. For the reader who may want further details, consult [5].
} 
3. movevar600(Time,Level1-7)

Standard deviation of acceleration at 10-min intervals.

4. movevar3600(Time,Level1-7)

Standard deviation of acceleration at 1-h intervals.

5. positionChange600(Time,Level1-7)

Total travel distance of acceleration at 10-min intervals.

6. positionChange3600(Time,Level1-7)

Total travel distance of acceleration at 1-h intervals.

7. beforeState(Time1,Time2)

Time1 and Time 2 are consecutive times.

8. sameTimeZoneState(Time1,Time2)

10-min of Time1 are included in 1-h of Time2.

Level1-8 (or 7) represents the magnitude of the value. Since ILP cannot handle continuous values directly, we divided them into several ranks according to the magnitude of the value and converted them to predicates. Intuitively, the larger the value of Level, the larger the actual value. Note that we distribute Levels not by an absolute value but a deviation value. For example, if a deviation value of acceleration is greater than or equal to 45 and less than 50 , it is classified as Level 4.

\section{Experiment}

\subsection{Outline of our Experiment}

In this study, we collected raw sensor data from 31 cows in NAMIKI Dairy Farm Co. Ltd. The data was collected for approximately two months (February 2, 2018 to April 30, 2018), continuously for $24 \mathrm{~h}$ and stored in CSV files. The data from these files was used to calculate the features shown in Section 3.2. The number of positive and negative cases were 435 and 4836 , respectively. Using ILP, we created a training model and verified its accuracy. In addition, we examined the obtained rules to determine the rules that would predict the cow's delivery.

\subsection{Result of Cross-validation}

In this study, we conducted a five-fold cross-validation to verify the accuracy of our model. Five-fold cross-validation is a method for verifying and confirming the validity of the analysis. First, we divide the features into five subsets and one was used for testing and the others for training. Then, this procedure is repeated five times with multiple subsets for testing. Finally, we calculate the average of the five results to verify the accuracy of our model. Table 2 shows our result. It is very useful to be able to classify the delivery of cows with an accuracy of 56It is more effective than human prediction.

\subsection{Obtained Rules}

We show the all of obtained rules by ILP in Table 3. As we mentioned, obtained rules are written in terms of a formula of first-order predicate logic. For example, the rule at the top of 
Table 2: Evaluation Indicator

\begin{tabular}{|c|c|}
\hline Accuracy & $56.79 \%$ \\
\hline Precision & $9.6 \%$ \\
\hline Recall & $50.46 \%$ \\
\hline F Value & $16.13 \%$ \\
\hline
\end{tabular}

the table shows that when it is transitioning from the state, the average of acceleration is Level 4 and the standard deviation of acceleration is Level 2. For state B, the average of acceleration is Level 4 and the standard deviation of acceleration is Level 1, demonstrating that cows may deliver. According to the above rules, we can predict delivery of cows. In contrast to the ordinary machine learning model, our model explains the rules that can predict cow's delivery directly. It is considered that only more reasonable rules can be obtained by changing learning parameters.

Table 3: Generated Rules

\begin{tabular}{|c|c|c|}
\hline Included Pos. Cases & Included Neg. Cases & The Rule \\
\hline 10 & 34 & $\begin{array}{l}\text { moveave600(A, Level4) } \wedge \text { move- } \\
\text { var600 }(\mathrm{A}, \text { Level1 }) \wedge \text { beforeState }(\mathrm{A}, \mathrm{B}) \wedge \\
\text { moveave600 }(\mathrm{B}, \text { Level4 }) \wedge \text { movevar600 }(\mathrm{B}, \\
\text { Level2 })\end{array}$ \\
\hline 10 & 14 & $\begin{array}{l}\text { movevar600(A, Level1) } \wedge \text { sameTimeZon- } \\
\text { eState }(\mathrm{A}, \mathrm{B}) \wedge \text { moveave3600(B, Level5) } \\
\wedge \text { movevar3600(B, Level1 }) \wedge \text { position- } \\
\text { Change3600(B, Level2) }\end{array}$ \\
\hline 14 & 29 & $\begin{array}{l}\text { sameTimeZoneState }(\mathrm{A}, \\
\text { moveave3600(B, Level5) }\end{array} \begin{array}{l}\mathrm{B}) \\
\text { var3600(B, Level1) } \\
\text { Change3600(B, Level2) }\end{array}{ }^{\wedge} \begin{array}{r}\text { move- } \\
\text { position- }\end{array}$ \\
\hline 11 & 49 & $\begin{array}{l}\text { moveave } 600(\mathrm{~A}, \text { Level3 }) \wedge \text { beforeState }(\mathrm{A}, \\
\mathrm{B}) \wedge \text { sameTimeZoneState }(\mathrm{A}, \quad \mathrm{C}) \wedge \\
\text { moveave3600 }(\mathrm{C}, \text { Level3 }) \wedge \text { moveave } 600(\mathrm{~B}, \\
\text { Level4 }\end{array}$ \\
\hline 11 & 49 & $\begin{array}{l}\text { beforeState }(\mathrm{A}, \mathrm{B}) \wedge \text { sameTimeZoneS- } \\
\text { tate }(\mathrm{A}, \mathrm{C}) \wedge \text { moveave3600 }(\mathrm{C}, \text { Level3 }) \wedge \\
\text { moveave600(B, Level4 }) \wedge \text { moveave600 }(\mathrm{A}, \\
\text { Level3) }\end{array}$ \\
\hline 12 & 35 & $\begin{array}{l}\text { movevar600(A, Level2 }) \wedge \text { sameTimeZon- } \\
\text { eState }(A, B) \wedge \text { moveave3600 }(B, \text { Level2 }) \wedge \\
\text { positionChange3600 }(B, \text { Level1 })\end{array}$ \\
\hline 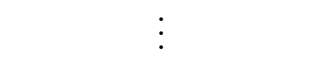 & $\vdots$ & $\vdots$ \\
\hline
\end{tabular}

We give the confusion matrix in Table 4. In this table, the horizontal and vertical axis indicate the actual label (true or false) and the predicted label by ILP, respectively. Each value in the table shows the number of applicable data values. For example, given an applicable data, 
if the actual label is true but predicted label by ILP is false, it is indicated in the lower left element of the table.

Table 4: Confusion Matrix

\begin{tabular}{|c|c|c|}
\hline \multicolumn{3}{|c|}{ Predicted Label } \\
\hline & True & False \\
\hline True & 219 & 215 \\
\hline False & 2062 & 2774 \\
\hline
\end{tabular}

However, as the accuracy is still low, we will improve the classification accuracy by adding new features and introduce applicable predicates using other machine learning methods in further studies.

\section{Conclusion and Future Works}

Our aim was to detect the cows' delivery time automatically with the use of machine learning. We collected raw sensor data from 31 cows, made features and labeled them for binary classification. Finally, we trained a model by ILP and verified the accuracy of the model. We also generated a set of readable rules that could predict the delivery of a cow. The resultant accuracy was $56.79 \%$ and we obtained 130 rules. In future studies, we plan on improving the accuracy by creating new features, introducing new predicates, and using other machine learning methods.

\section{References}

[1] DZ Caraviello, KA Weigel, M Craven, D Gianola, NB Cook, KV Nordlund, PM Fricke, and MC Wiltbank. Analysis of reproductive performance of lactating cows on large dairy farms using machine learning algorithms. Journal of dairy science, 89(12):4703-4722, 2006.

[2] Yoav Freund and Llew Mason. The alternating decision tree learning algorithm. In icml, volume 99, pages 124-133, 1999.

[3] Wilhelm Grzesiak, Piotr Błaszczyk, and René Lacroix. Methods of predicting milk yield in dairy cows - predictive capabilities of wood's lactation curve and artificial neural networks (anns). Computers and electronics in agriculture, 54(2):69-83, 2006.

[4] Nazira Mammadova and İsmail Keskin. Application of the support vector machine to predict subclinical mastitis in dairy cattle. The Scientific World Journal, 2013.

[5] Stephen Muggleton and Luc De Raedt. Inductive logic programming: Theory and methods. The Journal of Logic Programming, 19:629-679, 1994.

[6] Oliver Nelles, Alexander Fink, and Rolf Isermann. Local linear model trees (lolimot) toolbox for nonlinear system identification. IFAC Proceedings Volumes, 33(15):845-850, 2000.

[7] Saleh Shahinfar, Hassan Mehrabani-Yeganeh, Caro Lucas, Ahmad Kalhor, Majid Kazemian, and Kent A Weigel. Prediction of breeding values for dairy cattle using artificial neural networks and neuro-fuzzy systems. Computational and mathematical methods in medicine, 2012.

[8] Tomonobu Ozaki Yasuichi Kogawa and Ken Ueno. Inductive Logic Programming. Kyoristu Shuppan, 2001. 